\title{
The association of hyperglycaemia with prevalent tuberculosis: a population-based cross-sectional study
}

Sarah Lou Bailey ${ }^{1,2^{*}}$ (D) , Helen Ayles ${ }^{1,2}$, Nulda Beyers ${ }^{3}$, Peter Godfrey-Faussett ${ }^{1}$, Monde Muyoyeta ${ }^{2}$, Elizabeth du Toit ${ }^{3}$, John S. Yudkin ${ }^{4}$ and Sian Floyd ${ }^{5}$

\begin{abstract}
Background: Systematic reviews suggest that the incidence of diagnosed tuberculosis is two- to- three times higher in those with diabetes mellitus than in those without. Few studies have previously reported the association between diabetes or hyperglycaemia and the prevalence of active tuberculosis and none in a population-based study with microbiologically-defined tuberculosis. Most have instead concentrated on cases of diagnosed tuberculosis that present to health facilities. We had the opportunity to measure glycaemia alongside prevalent tuberculosis. A focus on prevalent tuberculosis enables estimation of the contribution of hyperglycaemia to the population prevalence of tuberculosis.
\end{abstract}

Methods: A population-based cross-sectional study was conducted among adults in 24 communities from Zambia and the Western Cape (WC) province of South Africa. Prevalent tuberculosis was defined by the presence of a respiratory sample that was culture positive for M. tuberculosis. Glycaemia was measured by random blood glucose (RBG) concentration. Association with prevalent tuberculosis was explored across the whole spectrum of glycaemia.

Results: Among 27,800 Zambian and 11,367 Western Cape participants, 4,431 (15.9\%) and 1,835 (16.1\%) respectively had a RBG concentration $\geq 7.0 \mathrm{mmol} / \mathrm{L}$, and $405(1.5 \%)$ and $322(2.8 \%)$ respectively had a RBG concentration $\geq 11$. $1 \mathrm{mmol} / \mathrm{L}$. In Zambia, the prevalence of tuberculosis was $0 \cdot 5 \%(142 / 27,395)$ among individuals with RBG concentration <11.1 mmol/L and also $\geq 11.1 \mathrm{mmol} / \mathrm{L}(2 / 405)$; corresponding figures for WC were $2 \cdot 5 \%(272 / 11,045)$ and $4 \cdot 0 \%$ (13/322). There was evidence for a positive linear association between hyperglycaemia and pulmonary prevalent tuberculosis. Taking a RBG cut-off $11.1 \mathrm{mmol} / \mathrm{L}$, a combined analysis of data from Zambian and WC communities found evidence of association between hyperglycaemia and TB (adjusted odds ratio $=2 \cdot 15,95 \% \mathrm{Cl}$ [1 17-3 94]). The population attributable fraction of prevalent tuberculosis to hyperglycaemia for Zambia and WC combined was $0.99 \%$ (95\% Cl 0.12\%-1.85\%) for hyperglycaemia with a RBG cut-off of $11.1 \mathrm{mmol} / \mathrm{L}$.

Conclusions: This study demonstrates an association between hyperglycaemia and prevalent tuberculosis in a large population-based survey in Zambia and Western Cape. However, assuming causation, this association contributes little to the prevalence of TB in these populations.

Keywords: Zambia, South Africa, Logistic regression

* Correspondence: slbailey@doctors.org.uk

'LSHTM TB Centre and Department of Clinical Research, London School of Hygiene and Tropical Medicine, London, UK

2ZAMBART Project, Lusaka, Zambia

Full list of author information is available at the end of the article International License (http://creativecommons.org/licenses/by/4.0/), which permits unrestricted use, distribution, and reproduction in any medium, provided you give appropriate credit to the original author(s) and the source, provide a link to the Creative Commons license, and indicate if changes were made. The Creative Commons Public Domain Dedication waiver (http://creativecommons.org/publicdomain/zero/1.0/) applies to the data made available in this article, unless otherwise stated. 


\section{Background}

The number of adults with diabetes mellitus globally is predicted to rise from 382 million in 2013 to 592 million in 2035 [1-6]. Of those in the world who currently have diabetes, four out of five live in a low or middle income country [6]. The burden of the anticipated rise in diabetes prevalence will fall largely to these low and middle income countries, the same countries that have some of the highest burdens of tuberculosis (TB) worldwide [4, 7].

Associations between diabetes and tuberculosis are increasingly recognised: systematic reviews and metaanalyses suggest that the incidence of active diagnosed tuberculosis is two- to- three times higher in those with diabetes compared to those without diabetes; [8-10] that diabetes increases the risk of death from diagnosed tuberculosis [11], and that diabetes may increase the risk of tuberculosis relapse [9, 11]. Studies from Africa have shown even stronger associations between diabetes and active diagnosed TB; a four-fold increase in diabetes prevalence was seen in TB patients in Dar es Salaam compared to the general population; [12] an odds ratio for $\mathrm{TB}$ of $8 \cdot 33$ was seen in the Congo comparing those with diabetes to those without diabetes [13]. As the prevalence of diabetes rises in locations with a high burden of tuberculosis, a deeper understanding of these associations is increasingly important.

We had the opportunity to measure glycaemia alongside tuberculosis in a population-based cross-sectional survey, which took place as part of the ZAMSTAR (Zambia South Africa TB and HIV Reduction Study) trial [14-16]. This trial was a $2 \times 2$ factorial community randomised trial to evaluate the impact of two complex interventions on the prevalence of $\mathrm{TB}$ in high HIV prevalence settings in Zambia and South Africa. The primary outcome for the study was the prevalence of tuberculosis after three years of intervention, measured through a cross-sectional survey of a random sample of adults from each community. We took this opportunity to also explore the association between hyperglycaemia and prevalent TB.

Both Zambia and South Africa have national TB control programmes, with a structured approach to TB diagnosis, management and control. Sputum smear microscopy is used for the diagnosis of pulmonary TB in Zambia. Few health care centres have access to culture or molecular tests. In South Africa Xpert MTB/RIF was introduced in 2011 for the diagnosis of pulmonary $\mathrm{TB}$, though interruption to the supply of cartridges has been a challenge. Supply of TB medication rarely suffers interruption and is provided free of charge to patients in both countries. The situation for diabetes management is less favourable. In Zambia, point-of-care glucometers are most commonly used for diagnosis, though frequently glucometer strips are unavailable. Metformin and glibenclamide are widely available but access to alternative oral hypoglycaemics can be challenging. Insulin is available but can be difficult to access in remote areas. Storage of insulin is frequently problematic for patients due to lack of access to refrigeration. Unlike for TB, diabetes medication is not provided to patients for free.

Few studies have previously reported the association between diabetes or hyperglycaemia and prevalent tuberculosis in the general population, and to our knowledge none in a population-based study with microbiologically-defined tuberculosis. Most have concentrated on cases of tuberculosis that present to, and are diagnosed, at health facilities. Two historical studies did measure the prevalence of tuberculosis in the general population; one in Philadelphia, USA in 1946 [17], the other in Kristianstad, Sweden in 1954 [18]. Both identified pulmonary TB by chest radiograph, and identified diabetes through referrals from clinics and through medical records respectively. Rather than being based in a general population, other studies that have focused on tuberculosis prevalence have investigated the prevalence among patients with diabetes in a clinic setting or identified through medical records, using either no comparison group or clinic patients without diabetes as a comparison group [10, 19, 20]. Exploration of the association of hyperglycaemia with prevalent tuberculosis in a population-based study allows for estimation of the contribution of hyperglycaemia to the population prevalence of tuberculosis.

The aims of this study are therefore to determine the association between hyperglycaemia and prevalent tuberculosis and to estimate the population attributable fractions of prevalent tuberculosis to hyperglycaemia, assuming causation, within our study communities in Zambia and the Western Cape region of South Africa.

\section{Methods}

This population-based cross-sectional study was nested within a $2 \times 2$ factorial cluster-randomised trial (the ZAMSTAR study [14-16]) and undertaken between January and December 2010 in 24 study communities: 16 from 5 provinces in Zambia and 8 from the Western Cape province of South Africa. Within each community, a two-stage cluster sampling design was used to recruit participants. Exclusion criteria were age $<18$ years, inability to give informed consent due to disability/incapacitation, refusal to submit a respiratory sample and any persons living in institutional settings.

Each participant was required to give written informed consent. Individuals and household heads were interviewed in their homes using structured questionnaires. Each participant was requested to produce a spot respiratory sample for tuberculosis culture. Finger prick capillary blood was taken for HIV testing and random blood glucose (RBG) measurement, with pre- and post-test counselling 
for HIV tests. RBG concentration was measured using an Optium Xceed point-of-care glucometer. All individuals identified to have abnormal blood glucose or to be HIV positive were referred to existing local health facilities for appropriate management.

Data were electronically entered directly onto personal digital assistants by field staff at the time of data collection, using pre-programmed questionnaires and result sheets. All information was downloaded daily into a SQL (structured query language) database and later exported into Stata.

All procedures for sputum sample collection and culture were identical in all study sites. Research staff in all sites were trained to instruct participants on adequate expectoration to achieve a lower rather than upper airways sample. Samples were collected daily from field sites and delivered to the laboratory in each study community. A standard liquid culture technique was used to isolate Mycobacterium tuberculosis (MGIT, Becton Dickinson). Growth detected by culture was identified using an immunochromatographic assay (Capilia TB), and all Capilia TB assay positive cultures were confirmed by $16 \mathrm{~S}$ ribosomal RNA sequencing. Detailed methods are described by Ayles et al. in the final report of the ZAMSTAR trial [16].

The Optium Xceed glucometer uses a whole blood capillary sample but is calibrated to report the plasma equivalent result. The results presented here are therefore the plasma equivalent glucose concentrations. This device was chosen because of its documented accuracy in multiple independent studies combined with its availability in Zambia and South Africa. It was found to be one of the most superior glucometers in all published accuracy studies, with between $84 \%$ and $100 \%$ of the results from the finger prick capillary specimen being within the recommended limits compared to reference plasma estimation on laboratory analysers [21-27]. When inaccuracy was seen, this was mostly for low rather than high glucose concentrations [24, 25, 27]. All research staff were trained on the use of this particular glucometer and were required to undergo proficiency testing. Standardised control solution was used for performance checks on test strips and meters.

Ethics approval was granted from the London School of Hygiene and Tropical Medicine Ethics Committee, the University of Stellenbosch Human Research Ethics Committee and the University of Zambia Biomedical Research Ethics Committee.

\section{Definitions}

- Hyperglycaemia, the exposure of interest for this study, is initially examined with RBG concentration as an ordered categorical variable. We then use sequential RBG cut-offs $-7.0 \mathrm{mmol} / \mathrm{L}, 7.8 \mathrm{mmol} / \mathrm{L}$, $9.0 \mathrm{mmol} / \mathrm{L}$ and $11.1 \mathrm{mmol} / \mathrm{L}$ - to explore increasing levels of hyperglycaemia. We based our cut-off levels on the current World Health Organisation guidelines for diabetes diagnosis and monitoring [28], though this was only to allow for exploration of increasing levels of glycaemia and not intended to be indicative of diabetes diagnoses.

- Prevalent pulmonary tuberculosis, the outcome of interest for this study, is defined by the presence of a respiratory sample that is culture positive for Mycobacterium tuberculosis.

- HIV status is defined by a combination of blood sampling plus self-report for those with missing biological data.

\section{Analysis strategy}

Principal components analysis was used to create a measure of household socio-economic position separately for each country. Unadjusted and adjusted odds ratios of the association between hyperglycaemia and prevalent tuberculosis were estimated using logistic regression analysis, accounting for within-cluster correlation resulting from the sampling design. Analyses were performed separately for Zambian and South African data due to heterogeneity between the two distinct locations. This enabled us to control for confounding differently for each location, which was necessary because of the big differences between the two settings. Fixedeffects meta-analysis of adjusted odds ratios from each country was then performed, with weights according to the inverse variance method to give overall odds ratios of the association between hyperglycaemia and prevalent tuberculosis. Population attributable fractions (PAFs) of prevalent tuberculosis to hyperglycaemia and HIV were calculated separately for each country and for combined estimates. These were calculated using the formula $P A F$ $=\sum p_{k}{ }^{\prime}\left(\theta_{k}-1\right) / \theta_{k}$ where $\mathrm{p}^{\prime}$ is the proportion of cases exposed in the study population at exposure level $k$ and $\theta$ is the adjusted odds ratio. Given that $\theta$ and p' were estimated from the same data, the $95 \%$ confidence intervals could be calculated using the following error factor for (1-PAF):

$$
\text { Error factor }=\exp \left\{1.96 \times \sqrt{\frac{n_{1} p^{\prime 2} V+2 p^{\prime}(\theta-1)+p^{\prime}\left(1-p^{\prime}\right)(\theta-1)^{2}}{n_{1}\left[\theta\left(1-p^{\prime}\right)+p^{\prime}\right]^{2}}}\right\}
$$

where $n_{1}$ is the total number of cases observed and V the variance of the adjusted log odds ratio (the standard error of the log odds ratio squared). Evidence for effect modification by gender and HIV was explored. All data analyses were performed using Stata 13. 


\section{Results}

The cross-sectional survey enrolled 57,809 (70.8\% of eligible) participants from 31,300 ( $88 \cdot 6 \%$ of eligible) households in Zambia and 32,792 (77.7\% of eligible) participants from 17,095 (85.3\% of eligible) households in Western Cape (Fig. 1). Evaluable sputum samples and complete RBG results were obtained for $27,800(48 \cdot 1 \%$ of enrolled) participants in Zambia and 11,367 (34.7\% of enrolled) participants in Western Cape. Data from these participants were analysed. Comparison of individuals with evaluable sputum samples with those with non-evaluable samples in Zambia showed them to be much the same (data presented as supplementary material to the ZAMSTAR trial publication) [16].

Among Zambian and Western Cape participants, $15.9 \%$ and $16.1 \%$ respectively had a RBG concentration $\geq 7.0 \mathrm{mmol} / \mathrm{L}$, and $1.5 \%$ and $2.8 \%$ respectively had a RBG concentration $\geq 11.1 \mathrm{mmol} / \mathrm{L}$. The prevalence of tuberculosis was approximately 500 per 100,000 $(0 \cdot 5 \%)$ among Zambian participants and approximately 2,500 per $100,000(2 \cdot 5 \%)$ among Western Cape participants. Tuberculosis prevalence stratified by glycaemia and baseline characteristics is shown in Tables 1 and 2.

Among individuals included in the analysis, HIV status was determined by blood sampling for 23,067 $(90 \cdot 2 \%)$ participants and by self-reported status for 2,501 (9.8\%) participants in Zambia. Corresponding values for Western Cape participants are 10,106 (94.8\%) and 551 (5.2\%). Among participants with RBG results, 2,232 participants in Zambia and 710 in Western Cape had missing data for both blood sampling and self-reported HIV status.

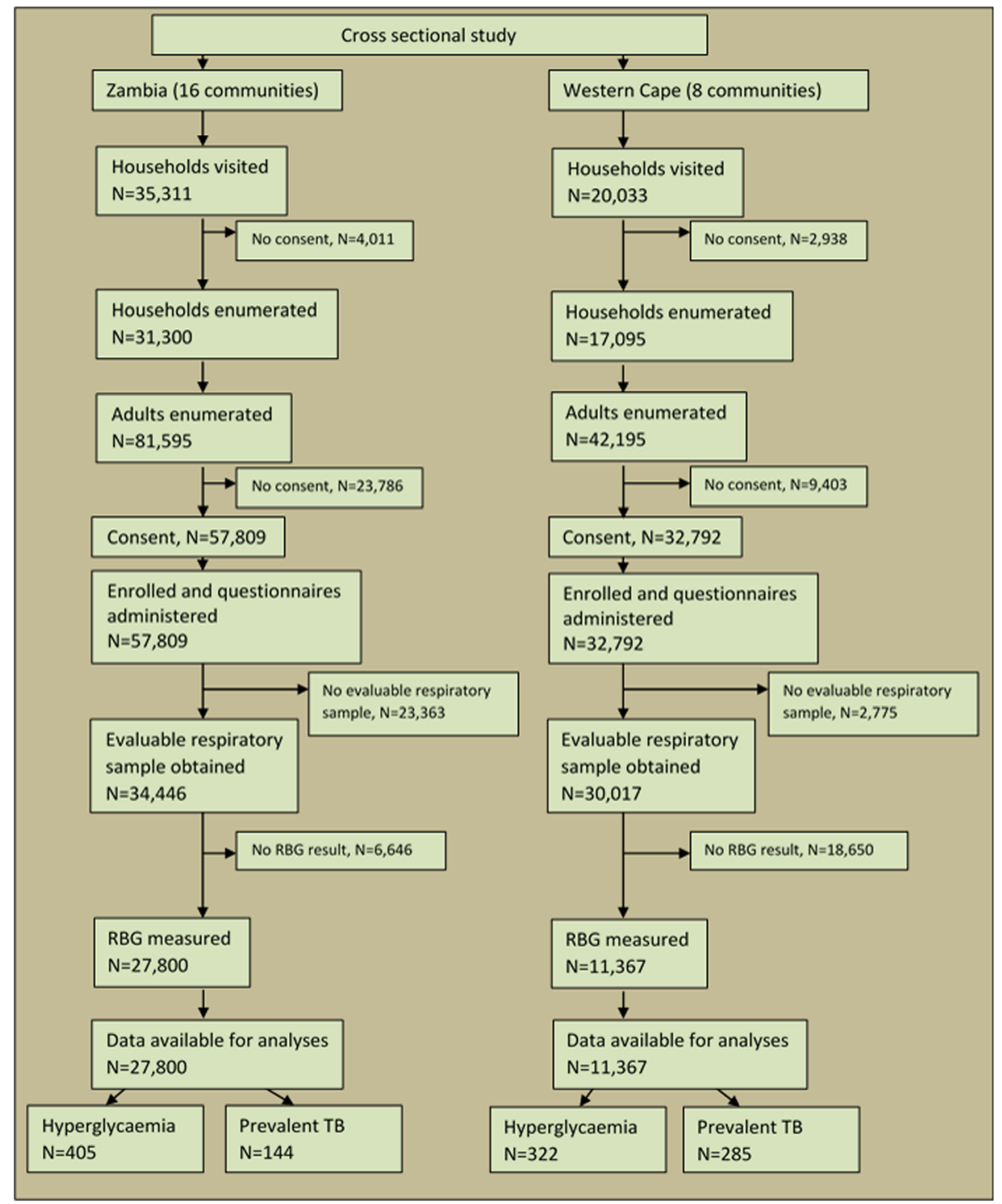

Fig. 1 Number and flow of participants and cases in this cross sectional study in Zambia and the Western Cape of South Africa 


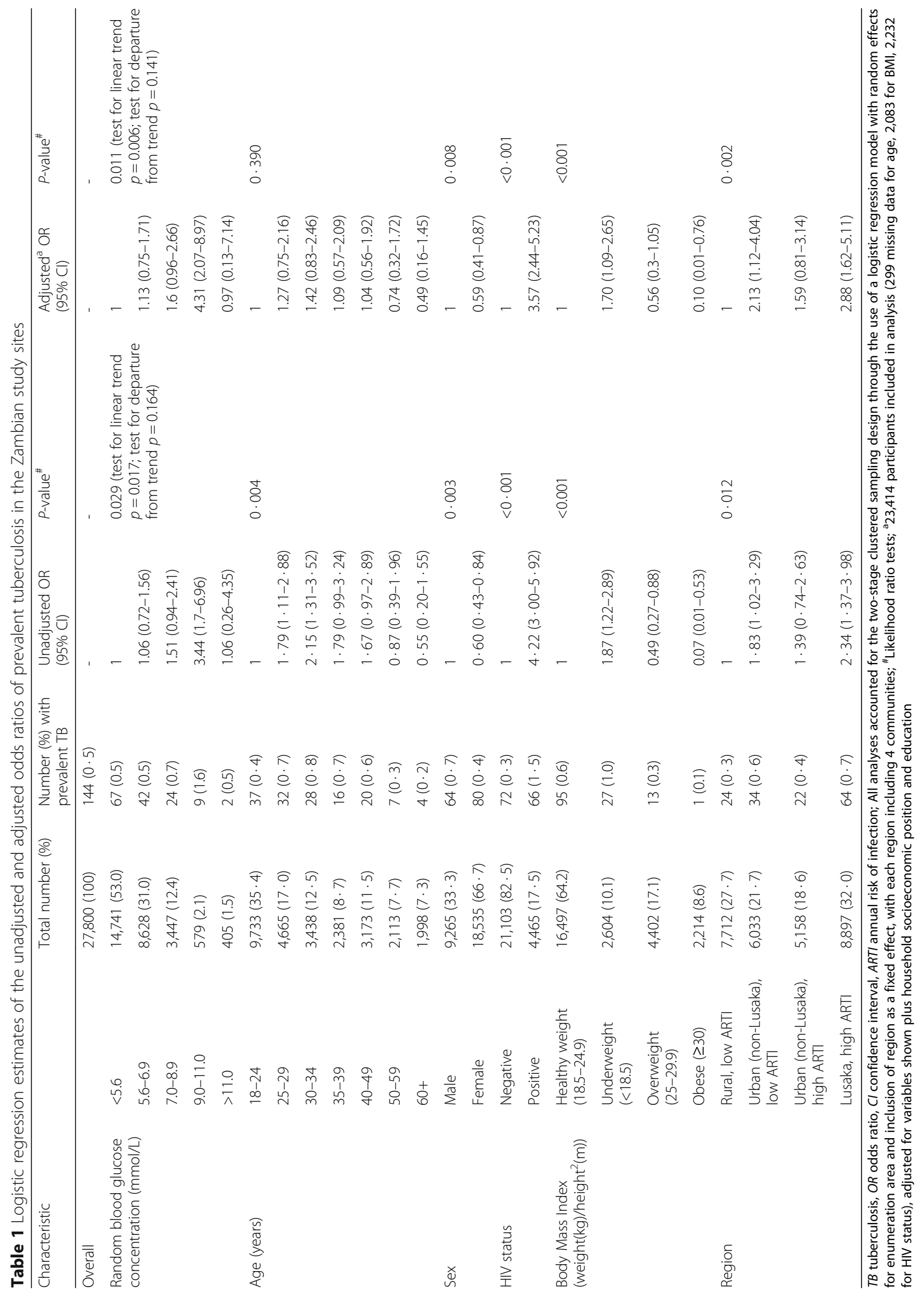




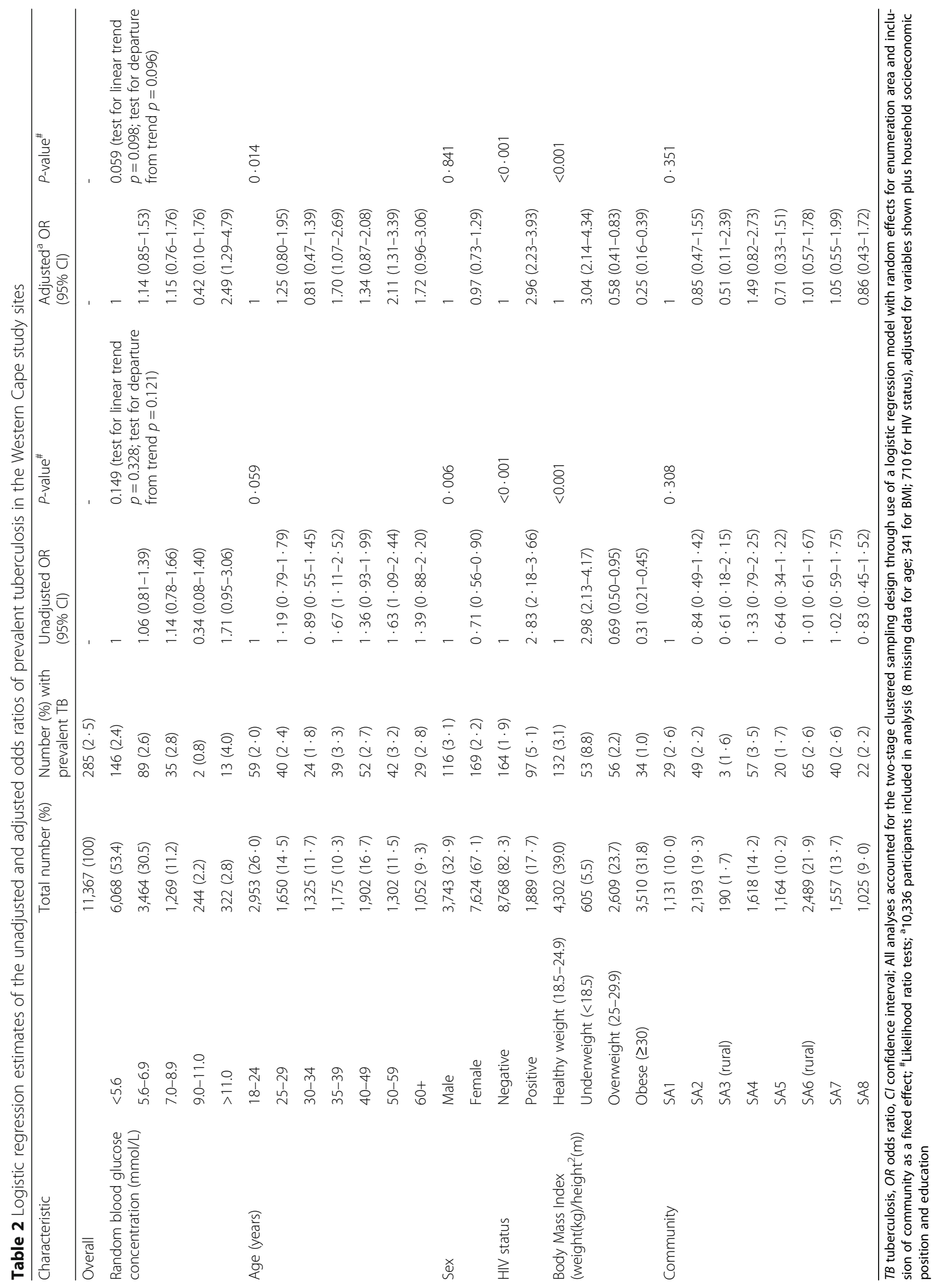


Among Zambian participants, as RBG concentration increased, the unadjusted and adjusted odds of prevalent TB initially increased compared to the baseline RBG concentration $<5.6 \mathrm{mmol} / \mathrm{L}$, peaking at RBG concentration 9.0$11.0 \mathrm{mmol} / \mathrm{L}$ (adjusted OR 4.31, 95\% CI [2.07-8.97]). Although the odds of prevalent $\mathrm{TB}$ did not continue to increase with increasing RBG concentration beyond this, the number of individuals with a RBG concentration $\geq 11.1 \mathrm{mmol} / \mathrm{L}$ was low and the confidence interval was wide so there was still strong evidence of a linear association between RBG concentration and TB prevalence $(p=0.006)$ after adjusting for age, sex, household socioeconomic position, education, body mass index, HIV status and geographical location (Table 1).

On multivariable analysis there was weak evidence of a linear association between glucose concentration and TB prevalence among Western Cape participants $(\mathrm{p}=0.06)$. In this location the adjusted odds of prevalent TB compared to the baseline was greatest for individuals with a RBG concentration $\geq 11.1 \mathrm{mmol} / \mathrm{L}$ (OR 2.49, 95\% CI [1.29-4.79], Table 2).

Unadjusted and adjusted odds ratios for prevalent TB using sequential RBG cut-offs to give increasing levels of hyperglycaemia are shown in Table 3 . The findings from Zambia and Western Cape, combined with fixed-effects meta-analyses, showed increasing adjusted odds of prevalent tuberculosis for increasing cut-off levels of hyperglycaemia, though the increase was small; and, across successive cut-off levels, confidence intervals overlapped (Table 4). There was no evidence that the adjusted odds ratios differed between Zambia and Western Cape (Table 4).

The evidence for association between hyperglycaemia and prevalent tuberculosis strengthened from univariable to multivariable analyses in both Zambia and the Western Cape communities. In the Zambian communities the predominant negative confounding factors were body mass index and HIV status. The Western Cape communities had the same negative confounding factors, while the predominant positive confounding factor was age.

On combined analysis, there was evidence of a contribution of hyperglycaemia to the population prevalence of tuberculosis throughout the spectrum of hyperglycaemia (Table 4). However, the PAFs of prevalent TB to hyperglycaemia were small, particularly for the higher RBG cut-offs. For RBG concentration $\geq 11.1 \mathrm{mmol} / \mathrm{L}$ the PAF of prevalent TB was 0.99\%, 95\% CI [0.12-1.85]. When analysed as separate locations, the confidence intervals for the PAFs were wide and showed less evidence for a contribution of hyperglycaemia to the population prevalence of tuberculosis in both Zambia and Western Cape (Table 3).

When stratified by age, for the highest RBG cut-off, the PAF of prevalent TB to hyperglycaemia increased with increasing age in Western Cape, reflecting the higher prevalence of hyperglycaemia in older age groups (Table 5). In Zambia, for this highest RBG cut-off $\geq 11.1 \mathrm{mmol} / \mathrm{L}$, there remained little evidence for a contribution of hyperglycaemia to the population prevalence of tuberculosis despite the rising prevalence of hyperglycaemia with increasing age, though confidence intervals were wide (Table 5).

For purposes of comparison to HIV, the PAF of prevalent TB to HIV was $12.72 \%, 95 \%$ CI [7.70-17.47] in the Zambian communities, and 11.72\%, 95\% CI [8.25-15.06] in the Western Cape communities.

For purposes of comparison to self-reported known diabetes, of individuals with a RBG $<11.1 \mathrm{mmol} / \mathrm{L}, 1.7 \%$ in Zambian and $6.8 \%$ in Western Cape communities reported having a previous diagnosis of diabetes, and $0.3 \%$ and $2.0 \%$ respectively reported being on treatment for diabetes. Of individuals with a RBG $\geq 11.1 \mathrm{mmol} / \mathrm{L}, 27.9 \%$ in Zambian and $57.5 \%$ in Western Cape communities reported having a previous diagnosis of diabetes, and $24.0 \%$ and $49.1 \%$ respectively reported being on treatment for diabetes. Incorporating participants with self-reported previously diagnosed diabetes into the highest category of hyperglycaemia made little difference to the odds ratio point estimates in Zambia and reduced the association seen with RBG concentration $\geq 11.1 \mathrm{mmol} / \mathrm{L}$ in Western Cape towards the null. Defining diabetes by self-report, the odds of prevalent TB for individuals with diabetes compared to those without was 0.83 , 95\% CI [0.20-3.46] in Zambia and 0.76, 95\% CI [0.44-1.32] in Western Cape. For individuals in Western Cape with self-reported diabetes and RBG $\geq 11.1 \mathrm{mmol} / \mathrm{L}$ the odds of prevalent TB was $1.55,95 \%$ CI [0.60-4.01] compared to individuals who did not report diabetes. For those with self-reported diabetes and RBG $<11.1 \mathrm{mmol} / \mathrm{L}$ this odds ratio was 0.61 , 95\% CI [0.32-1.18].

Subgroup analyses were not possible for the Zambian data using the highest $R B G$ cut-off $\geq 11.1 \mathrm{mmol} / \mathrm{L}$ due to limited data, and no difference was seen for gender and HIV categories using the RBG cut-off $\geq 9.0 \mathrm{mmol} / \mathrm{L}$. In the Western Cape using the RBG cut-off $\geq 11.1 \mathrm{mmol} / \mathrm{L}$, the point estimate of the adjusted odds of hyperglycaemia on prevalent tuberculosis was higher among women than men (for men $\mathrm{OR}=1 \cdot 92$, 95\% CI $[0 \cdot 55-6 \cdot 68]$; for women $\mathrm{OR}=2 \cdot 58,95 \% \mathrm{CI}[1 \cdot 24-5 \cdot 35]$; but there was no evidence the odds ratio differed for men and women, test for interaction $p=0 \cdot 68$ ). It was higher among those with HIV than those without HIV (among those with HIV $\mathrm{OR}=5 \cdot 34$, 95\% CI $[1 \cdot 56-18 \cdot 23]$; among those without HIV OR $=1 \cdot 90,95 \%$ CI $[0 \cdot 89-4 \cdot 04]$; but the evidence for interaction was weak $(p=0 \cdot 17))$.

\section{Discussion}

This is the first ever population based study of prevalent tuberculosis diagnosed microbiologically and glycaemia. 


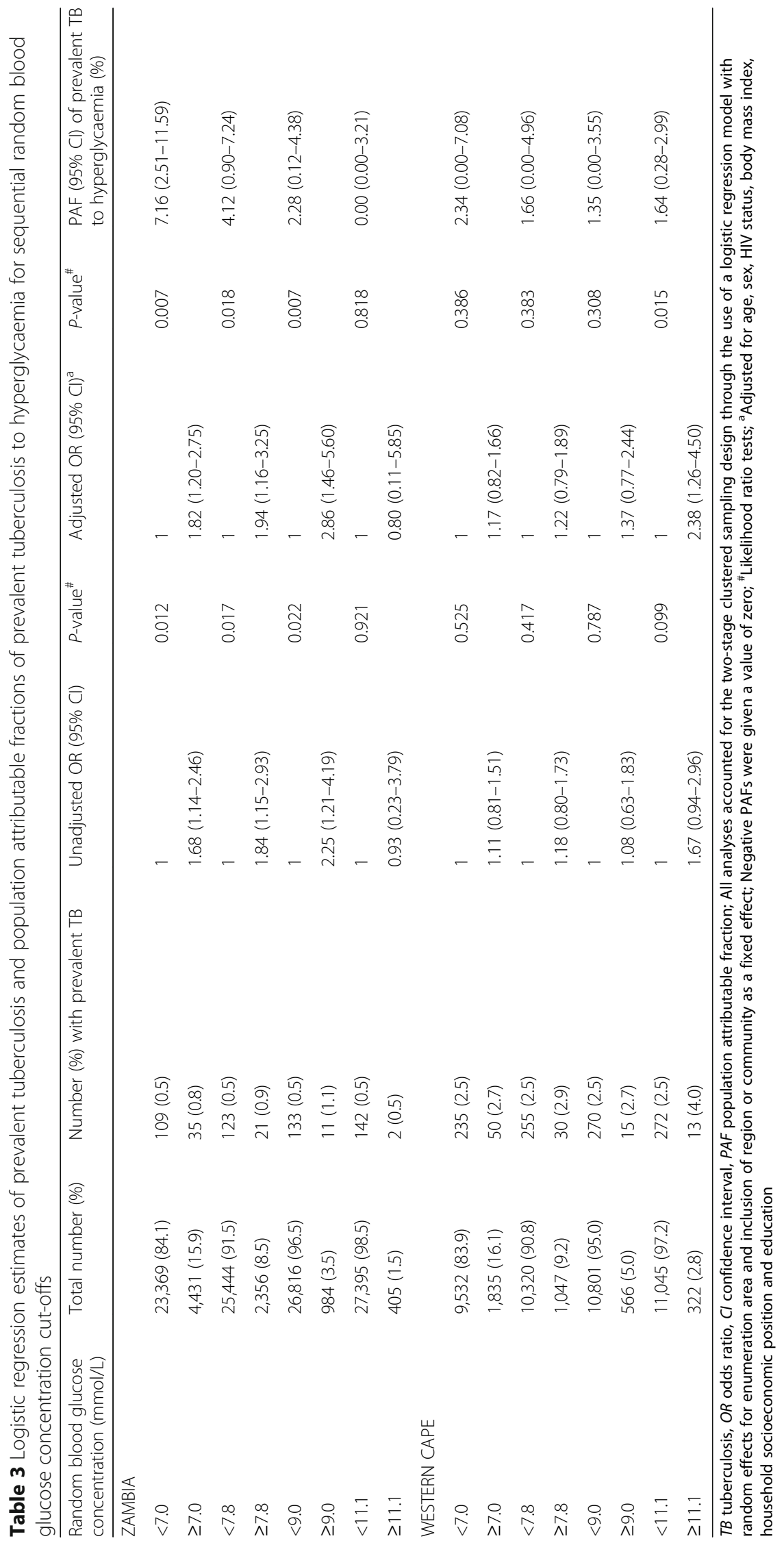


Table 4 Combined adjusted odds ratios of prevalent tuberculosis for Zambia and Western Cape and associated population attributable fractions of prevalent tuberculosis to hyperglycaemia for sequential random blood glucose concentration cut-offs

\begin{tabular}{lllll}
\hline $\begin{array}{l}\text { Random blood glucose } \\
\text { concentration cut-off (mmol/L) }\end{array}$ & Combined adjusted OR* & P-value & $I^{2} p$-value & $\begin{array}{l}\text { PAF (95\% Cl) of prevalent TB } \\
\text { to hyperglycaemia (\%) }\end{array}$ \\
\hline 7.0 & $1.40(1.07-1.84)$ & 0.013 & 0.112 & $4.57(1.27-7.77)$ \\
7.8 & $1.48(1.06-2.07)$ & 0.020 & 0.176 & $2.82(0.64-4.95)$ \\
9.0 & $1.87(1.21-2.90)$ & 0.005 & 0.104 & $1.84(0.56-3.11)$ \\
11.1 & $2.15(1.17-3.94)$ & 0.013 & 0.306 & $0.99(0.12-1.85)$ \\
\hline
\end{tabular}

OR odds ratio, $\mathrm{Cl}$ confidence interval, PAF population attributable fraction; ORs combined through fixed-effects meta-analysis; " ${ }^{\prime}$ Likelihood ratio tests; All analyses accounted for the two-stage clustered sampling design through the use of a logistic regression model with random effects for enumeration area and inclusion of region or community as a fixed effect; Negative PAFs were given a value of zero; *Adjusted for age, sex, HIV status, body mass index, household socioeconomic position and education

We used participants who were randomly selected from the community rather than exploring the association among participants who had already been diagnosed with tuberculosis or hyperglycaemia. Among the Zambian participants of our study there was good evidence of a positive linear association between hyperglycaemia and prevalent tuberculosis, and weak evidence for the same association in Western Cape. When data from the two locations were combined, there was evidence of association between hyperglycaemia and prevalent pulmonary tuberculosis across the spectrum of hyperglycaemia. On combined analysis the odds of prevalent tuberculosis was greatest for individuals with the highest level of glycaemia, though the magnitude of association was small. Those with a RBG concentration $\geq 11.1 \mathrm{mmol} / \mathrm{L}$, had 2.15 times the odds of prevalent tuberculosis than those with a RBG concentration $<11.1 \mathrm{mmol} / \mathrm{L}$.

This association seen in the study communities between hyperglycaemia and prevalent tuberculosis is consistent with the association seen elsewhere in the world between diabetes and prevalent TB $[17,18]$ and also between diabetes and active diagnosed TB [8-10].

Assuming causation, hyperglycaemia contributes little to the prevalence of tuberculosis throughout the spectrum of hyperglycaemia in the Zambian and Western Cape populations. This suggests that hyperglycaemia has only a small impact on the prevalence of TB in the study areas of Zambia and Western Cape despite the positive association seen between hyperglycaemia and prevalent TB in these locations. When stratified by age, however, we can see that the contribution of hyperglycaemia to the prevalence of tuberculosis in Western Cape is greater for older age groups, reflecting the higher prevalence of hyperglycaemia among older individuals. The same trend is seen in the Zambian study population, but the lower prevalence of hyperglycaemia in this setting means that confidence intervals are wide and point estimates remain low even for the oldest age groups.

The combined odds ratios were weighted towards the Western Cape estimates due to the larger number of individuals with hyperglycaemia and tuberculosis in this setting, particularly for RBG concentration $\geq 11.1 \mathrm{mmol} /$ L. Given the uncertainty of the Zambian odds ratio for this higher level of glycaemia the combined analyses yield the more reliable conclusions. However, regardless of which analysis is used, the conclusion remains the same, that the contribution of hyperglycaemia to the population prevalence of tuberculosis is low.

In this study we measured hyperglycaemia using a single RBG test. To optimise the accuracy of the test research

Table 5 Population attributable fraction of prevalent tuberculosis to hyperglycaemia for Zambian and Western Cape communities, stratified by age, using random blood glucose concentration cut-off $11.1 \mathrm{mmol} / \mathrm{L}$

\begin{tabular}{|c|c|c|c|c|}
\hline \multirow[t]{2}{*}{ Age (years) } & \multicolumn{2}{|l|}{ Zambia } & \multicolumn{2}{|l|}{ Western Cape } \\
\hline & Hyperglycaemia prevalence (\%) & PAF $(95 \% \mathrm{Cl})$ & Hyperglycaemia prevalence (\%) & PAF $(95 \% \mathrm{Cl})$ \\
\hline $18-24$ & 0.42 & $0.00(0.00-0.96)$ & 0.27 & $0.16(0.00-0.52)$ \\
\hline $25-29$ & 0.51 & $0.00(0.00-1.16)$ & 0.42 & $0.24(0.00-0.70)$ \\
\hline $30-34$ & 1.22 & $0.00(0.00-2.72)$ & 0.75 & $0.43(0.00-1.05)$ \\
\hline $35-39$ & 1.18 & $0.00(0.00-2.63)$ & 2.04 & $1.18(0.07-2.28)$ \\
\hline $40-49$ & 2.27 & $0.00(0.00-4.95)$ & 4.26 & $2.47(0.67-4.24)$ \\
\hline $50-59$ & 4.69 & $0.00(0.00-9.88)$ & 7.76 & $4.50(1.70-7.22)$ \\
\hline $60+$ & 4.60 & $0.00(0.00-9.70)$ & 8.65 & $5.01(1.97-7.97)$ \\
\hline Total & 1.45 & $0.00(0.00-3.21)$ & 2.83 & $1.64(0.28-2.99)$ \\
\hline
\end{tabular}

PAF population attributable fraction, $\mathrm{Cl}$ confidence interval; Hyperglycaemia defined as a random blood glucose concentration $\geq 11.1 \mathrm{mmol} / \mathrm{L}$; Negative PAFs were given a value of zero 
staff were carefully trained and tested, and the point-ofcare measure was carefully calibrated using standardised control solution, although not validated against laboratory glucose analyses. Use of this point-of-care test enabled glucose measurement of many participants in a large-scale field study located within the community. Use of a test that is more complicated to administer, such as a fasting blood glucose, oral glucose tolerance test or glycated haemoglobin, would have been logistically challenging and potentially less acceptable to participants, resulting in a much lower uptake of eligible participants and possibly introducing selection bias. Therefore, although not a test to diagnose diabetes, it was felt that the use of a RBG test was most likely to minimise overall bias and loss of study power in this setting of a large-scale population survey, spanning communities and countries. Therefore, rather than diagnosing diabetes, we have measured glycaemia and explored the effect of hyperglycaemia on tuberculosis prevalence. RBG tests normally have good specificity but sub-optimal sensitivity for diabetes $[29,30]$. A study in China found measurement of RBG concentration with a cut off of $11.1 \mathrm{mmol} / \mathrm{L}$ to have a sensitivity for diabetes of only $54 \cdot 8 \%$ compared to an oral glucose tolerance test. [31] This would suggest that association seen with hyperglycaemia based on RBG concentration would be an under-estimate of any association with diabetes. However, in the context of active TB disease the specificity of this test for diabetes could also be reduced, as those with stress-induced hyperglycaemia secondary to their TB disease could also have a high RBG concentration. This would result in an over-estimate of the association with TB based on RBG concentration, compared to association with diabetes. A final consideration is a single RBG measurement fails to give data on chronic hyperglycaemia, so we are unable to explore the effect of chronic hyperglycaemia on tuberculosis prevalence from these data. We did explore the effect of participants having previously diagnosed diabetes who may be on treatment and therefore may be normoglycaemic at the time of RBG testing but have longer term hyperglycaemia. The number of these participants were few in both Zambia and Western Cape and when incorporated into the categories of hyperglycaemia did not increase the odds ratio point estimates of the association with prevalent TB in either study location. Regardless of whether the hyperglycaemia measured was due to diabetes, was a consequence of TB disease or was transient from any other cause, the conclusion remains that in our study communities hyperglycaemia contributes little to the population prevalence of tuberculosis.

The substantial losses of evaluable respiratory samples in this study resulted largely from a failure of the positive mycobacterial control to grow in two of the laboratories in Zambia, causing whole batches to be nonevaluable. This has resulted in reduced study power, but is unlikely to have introduced bias to the study results, as the process could not have been associated with the presence or absence of hyperglycaemia in the individuals affected by these missing data. The missing glycaemic data is similarly unlikely to produce bias and was probably the consequence of the lack of prioritisation during data collection, a consequence of nesting this study within a larger cluster-randomised trial. Therefore, this too is unlikely to have been associated with the presence or absence of disease and so is also unlikely to have introduced substantial bias.

The participants in this study were randomly selected from their communities and so are representative of the general population within each community. The communities included were from urban and peri-urban settings and so rural populations are under-represented in this study. The prevalence of both hyperglycaemia and tuberculosis would therefore likely be lower in a general population sample.

In subgroup analysis, the association between hyperglycaemia and prevalent TB among those with HIV was stronger than among those without HIV, which could suggest that hyperglycaemia and HIV work synergistically to increase one's risk of TB, or could instead reflect an increase in stress-induced hyperglycaemia among those with HIV compared to those without HIV. These findings should be seen as hypothesis generating as the evidence for effect modification was weak and our data are underpowered for formal assessment of effect modification.

\section{Conclusion}

In our study communities in Zambia and Western Cape, there is evidence for a positive linear association between hyperglycaemia and prevalent pulmonary tuberculosis. On combined analysis, individuals with RBG concentration $\geq 11.1 \mathrm{mmol} / \mathrm{L}$ had 2.15 times the odds of prevalent tuberculosis than individuals with a RBG concentration $<11.1 \mathrm{mmol} / \mathrm{L}$. Despite this, assuming causation, hyperglycaemia contributes little to the tuberculosis prevalence in our study communities. Investigation of the associations between hyperglycaemia, diabetes and active diagnosed tuberculosis in these study communities would be a valuable addition to the findings from this study, and would allow for sub-group analysis of association with smear-negative, smear-positive and drug-resistant tuberculosis.

\section{Abbreviations \\ Cl: Confidence interval; FBG: Fasting blood glucose; $\mathrm{HbA}_{1}$ : Glycated haemoglobin; HIV: Human immunodeficiency virus; OR: Odds ratio; PAF: Population attributable fraction; RBG: Random blood glucose; TB: Tuberculosis; WC: Western Cape}

\section{Acknowledgements}

We thank all the members of all study teams who contributed to data collection. We thank the Zambia Ministry of Health, City of Cape Town 
Health Directorate, the Western Cape Provincial Department of Health, and Zambian and South African National TB Programmes and all the district health managers and health facility staff for allowing us to work in the facilities. We thank the Community Advisory Boards and the members of the community who participated in the trial. We acknowledge the support and scientific guidance of the CREATE consortium leadership, R Chaisson (principal investigator for CREATE) and L Moulton (coprincipal investigator for CREATE biostatistics core) and of the ZAMSTAR Study Advisory Group; M Borgdorff, D Enarson, B Chirwa, P Mwaba, Y Mulla, L Mvusi, M Poolman, I Toms and $\mathrm{K}$ Jennings.

\section{Funding}

SLB is supported by a Wellcome Trust Clinical PhD Fellowship (100141/Z/12/ Z). The ZAMSTAR study was supported by a subcontract from Johns Hopkins University with funds provided by grant number 19790.01 from the Bill and Melinda Gates Foundation. The contents of this manuscript are solely the responsibility of the authors and do not necessarily represent the official views of the Bill and Melinda Gates Foundation or the Wellcome Trust.

\section{Availability of data and materials}

Data are from the ZAMSTAR study whose authors may be contacted via Sian.Floyd@lshtm.ac.uk.

\section{Authors' contributions}

All authors contributed to initial study concept and study design. HA, NB and PGF are the principal investigators of the ZAMSTAR study, into which this study is nested. They oversaw participant recruitment and data collection. MM and EDT contributed to the management and supervision of data collection. SLB and SF performed the data analysis. SLB wrote initial drafts and all authors contributed to final editing of the paper. All authors read and approved the final manuscript.

\section{Competing interests}

The authors declare that they have no competing interests.

\section{Consent for publication}

Not applicable.

\section{Ethics approval and consent to participate}

Ethics approval was granted from the London School of Hygiene and Tropical Medicine Ethics Committee, the University of Stellenbosch Human Research Ethics Committee and the University of Zambia Biomedical Research Ethics Committee Each study participant was required to give written informed consent.

\section{Author details}

'LSHTM TB Centre and Department of Clinical Research, London School of Hygiene and Tropical Medicine, London, UK. ${ }^{2}$ ZAMBART Project, Lusaka, Zambia. ${ }^{3}$ Desmond Tutu TB Centre, Department of Paediatrics and Child Health, Stellenbosch University, Stellenbosch, South Africa. ${ }^{4}$ University College London, London, UK. ${ }^{5}$ LSHTM TB Centre and Department of Infectious Disease Epidemiology, London School of Hygiene and Tropical Medicine, London, UK

\section{Received: 21 June 2016 Accepted: 25 November 2016}

\section{Published online: 05 December 2016}

\section{References}

1. International Diabetes Federation. DF Diabetes Atlas. 6th ed. Belgium: International Diabetes Federation; 2013.

2. Mbanya JC, et al. Diabetes in sub-Saharan Africa. Lancet. 2010;375(9733):2254-66.

3. Miranda JJ, et al. Non-communicable diseases in low- and middle-income countries: context, determinants and health policy. Trop Med Int Health. 2008:13(10):1225-34.

4. Hall V, et al. Diabetes in Sub Saharan Africa 1999-2011: epidemiology and public health implications. A systematic review. BMC Public Health. 2011;11:564.

5. Danaei $\mathrm{G}$, et al. National, regional, and global trends in fasting plasma glucose and diabetes prevalence since 1980: systematic analysis of health examination surveys and epidemiological studies with 370 country-years and 2.7 million participants. Lancet. 2011;378(9785):31-40.

6. Guariguata L. By the numbers: new estimates from the IDF Diabetes Atlas Update for 2012. Diabetes Res Clin Pract. 2012;98(3):524-5.
7. Whiting DR, et al. IDF diabetes atlas: global estimates of the prevalence of diabetes for 2011 and 2030. Diabetes Res Clin Pract. 2011;94(3):311-21.

8. Jeon CY, Murray MB. Diabetes mellitus increases the risk of active tuberculosis: a systematic review of 13 observational studies. PLoS Med. 2008;5(7), e152.

9. Dooley KE, Chaisson RE. Tuberculosis and diabetes mellitus: convergence of two epidemics. Lancet Infect Dis. 2009;9(12):737-46.

10. Jeon CY, et al. Bi-directional screening for tuberculosis and diabetes: a systematic review. Trop Med Int Health. 2010;15(11):1300-14.

11. Baker MA, et al. The impact of diabetes on tuberculosis treatment outcomes: a systematic review. BMC Med. 2011;9:81.

12. Mugusi F, et al. Increased prevalence of diabetes mellitus in patients with pulmonary tuberculosis in Tanzania. Tubercle. 1990;71(4):271-6.

13. Mboussa J, et al. Course of pulmonary tuberculosis in diabetics. Rev Pneumol Clin. 2003:59(1):39-44

14. Ayles HM, et al. ZAMSTAR, The Zambia South Africa TB and HIV Reduction Study: design of a $2 \times 2$ factorial community randomized trial. Trials. 2008:9:63.

15. Sismanidis C, et al. Restricted randomization of ZAMSTAR: a $2 \times 2$ factorial cluster randomized trial. Clin Trials. 2008:5(4):316-27.

16. Ayles $\mathrm{H}$, et al. Effect of household and community interventions on the burden of tuberculosis in southern Africa: the ZAMSTAR communityrandomised trial. Lancet. 2013;382(9899):1183-94.

17. Boucot KR, et al. Tuberculosis among diabetics: the Philadelphia survey. Am Rev Tuberc. 1952;65(1:2):1-50.

18. Silwer $\mathrm{H}$, Oscarsson PN. Incidence and coincidence of diabetes mellitus and pulmonary tuberculosis in a Swedish county. Acta Med Scand Suppl. 1958:335:1-48.

19. Davidovich $D$, Aiello CR, Hassan IA. Antitubercular preventive examination in diabetics. Sem Med. 1963;122:781-4.

20. Marton S, et al. Representative Tuberculosis Mass Examinations in Diabetics in Hungary. Acta Tuberc Pneumol Scand. 1963;43:29-38.

21. Robinson CS, Sharp P. Tighter accuracy standards within point-of-care blood glucose monitoring: how six commonly used systems compare. J Diabetes Sci Technol. 2012;6(3):547-54.

22. Jday-Daly I, et al. [Multicenter evaluation of the reliability of five blood glucose monitoring systems]. Ann Biol Clin (Paris). 2011;69(1):55-61.

23. Kuo $C Y$, et al. Accuracy and precision evaluation of seven self-monitoring blood glucose systems. Diabetes Technol Ther. 2011;13(5):596-600.

24. Sonmez A, et al. The accuracy of home glucose meters in hypoglycemia. Diabetes Technol Ther. 2010;12(8):619-26.

25. Dimeski G, et al. Glucose meters: evaluation of the new formulation measuring strips from Roche (Accu-Chek) and Abbott (MediSense). Ann Clin Biochem. 2010;47(Pt 4):358-65.

26. Florkowski $\mathrm{C}$, et al. Comparison of blood glucose meters in a New Zealand diabetes centre. Ann Clin Biochem. 2009;46(Pt 4):302-5.

27. Coyne S, Lacour B, Hennequin-Le Meur C. Evaluation of Optium Xceed (Abbott) and One Touch Ultra (Lifescan) glucose meters. Ann Biol Clin (Paris). 2008:66(3):249-54.

28. World Health Organization. Definition and Diagnosis of Diabetes Mellitus and Intermediate Hyperglycemia: Report of a WHO/IDF Consultation. Geneva: World Health Organization; 2006.

29. Schneider H, Shaw J, Zimmet P. Guidelines for the detection of diabetes mellitus-diagnostic criteria and rationale for screening. Clin Biochem Rev. 2003:24(3):77-80.

30. World Health Organization. Definition, diagnosis and classification of diabetes mellitus and its complications. Geneva: World Health Organization; 1999.

31. Woo J, et al. The prevalence of diabetes mellitus and an assessment of methods of detection among a community of elderly Chinese in Hong Kong. Diabetologia. 1987;30(11):863-8. 